containing Group II serum and three marks those containing Group III serum. The tubes are stored away in boxes at room temperature but protected from the light. These tulics may be kept in the laboratory and are ready for immediate use whenever required. Serum preserved in this way retains its agglutinating power six months or longer.

With Group II and Group III scrum on hand, the determination of the group of any individual is easily and quickly donc. A single drop of blood from the finger-tip or ear is allowed to fall into a test tube containing 8 or 10 c.c. of physiologic sodium chlorid solution ( 0.85 per cent.). The tuhe is shaken or rotated in order to obtain a uniform suspension. On each of two cover slips a very small drop of the corpuscle suspension is placed by means of a capillary pipet or platinum loop; to one a droj of Group II serum is added and to the other a drop of Group III serum. The serum and corpuscles are mixed on each cover slip, inverted over a hollow ground slide, and examined uncler the microscope. $\Lambda$ gglutination may take place in a few minutes at room temperature, but it is a safe rule to allow half an hour at $37 \mathrm{C}$. to elapse before concluding that there is no agglutination.

\section{CHONDROMA OF THE SOFT PALATE, LONG OVERLOOKED}

Ben C. Gile, M.D., Puiladelpiija

It sounds like a paradox to say that we need to be reminded of familiar truths, but this is a fact. Every physician knows that a correct diagnosis is invaluable and that we should carnestly strive to discover and remove the cause of the diseases we encounter, and yet quite frequently we learn of cases in which the treatment has been exclusively symptomatic and etiologic investigation has been entirely neglected. - Of course, we are often required to treat symptoms: it may be our first duty to check a hemorrhage or relieve a severe pain, irrespective of causation; when we have time for investigation, however, especially in chronic cases, purely symptomatic treatment is justificd only by conditions in which: (1) the cause cannot be discovered; (2) the catise is a self-limiting process, as in many of the disorders occurring during pregnancy, or (3) the cause, though known, is beyond remedy and must be reckoned with as a permanent factor. Apart from situations such as these, the restriction of our therapy to the palliation of symptoms is negligence hard to condone.

In the case described hercin, a slowly growing tumor, in an accessible locality, had reached a considerable size and had given rise to a number of symptoms. The patient had been successively under the care of four practitioners, two of them laryngologists. Various remedial measures, medical and surgical, had been employed, but all treatment had been addressed to symptoms: no one did anything with the causal tumor and, in truth, only one of the four scrutinized the region in which it was to be found. The varied treatments temporarily palliated the symptoms, but had no permanent cffects, and the patient was growing worse until at last the tumor was recognized as the sole cause of the morbid conditions, and was extirpated, bringing about complete recovery.

As some of the statements in this narrative may seem strange, I wish to be particularly clear and definite regarding the authority on which they rest. Nothing which transpired before I took charge of the case came within my own personal knowledge. When the patient consulted me, she had already parted with her former advisers and I had no opportunity to confer with any of them. The patient is a young woman of intelligence, and I believe her to be entirely trustworthy. On account of her prolonged treatment and her large outlay of money, this illness was for her a very trying cpisode, and she put in writing a history of her experiences, from the time when she first sought medical aid until its final cessation, on her recovery of health. It is on this record that $I$ have depended for the facts preceding the time when I made her acquaintance. I believe that, to the best of her ability, she has told with accuracy what occurred in her case.
REPORT OF CASE

History.-In 1911, Miss E. W., aged about 30, sought the advice of her family physician, because of persistent earache on the right side. There was no discharge, and hearing was not impaired. The throat was not examined, and the treatment consisted of internal medication. The pain gradually passed away.

In 1913 there was another prolonged attack of earache and an intercurrent tonsillitis. Both subsided in a couple of weeks, but left a cough which continued for months. The same physician was in charge, and the patient took many bottles of medicine, the character of which she did not know. There were no surgical procedures.

In 1914 a very painful abscess developed in the right ear and was lanced by Dr. $X$, a laryngologist. No examination of throat or nose was made. Later in the same year occurred another attack of tonsillitis with troublesome cough. These affections were treated by Dr. Y, who administered a good deal of medicine by the stomach. No surgery was done, and no examination of the nose or of the nasopharynx was made.

Near the end of 1914, the patient, much discouraged by her frequent attacks of illness, applied to a laryngologist, Dr. Z, and was under his care for eight months, extending beyond the summer of 1915. There was no surgery, but medicines were applied to the throat and nostrils. With warm weather the symptoms were somewhat mitigated.

In Aprit, 1916, there was another abscess in the right ear, accompanied by a persistent cough. The patient returned to the laryngologist, Dr. $Z$, who lanced the alsscess and also inspected the soft palate, as far as that could be done by simply looking into the mouth without any instrumental aid, or any attempt to take a posterior view. Inadequate as this examination was, it showed that the soft palate was distended by tumefaction of some kind, and Dr, $Z$ informed the patient of the alnormal condition which existed, adding that he did not know what the growth was and did not think it wise to interfere with it. A Wassermann test was made with negative result; but the eustachian catheter was not used, nor were the Valsalva or Politzer methods of inflation. The patient continued to visit Dr. Z up to August, 1916, when swelling again appeared in the right ear and lancing brought temporary relief; but the trouble soon returned, and Miss W. in a discouraged frame of mind, was looking forward to another repetition of the incision, when the condition of a tooth led her to consult Dr. Briggs, a dentist in whom she had confidence. This was a fortunate incident, for Dr. Briggs assured her that the throat was seriously affected and required radical treatment to reach the cause of the recurrent disorder in the ear. At his solicitation she came to my office, Sept. 30, 1916.

Examination.-At that time, in addition to mental and physical depression, the patient was suffering from a rasping cough and her throat was sore, though not acutely painful. Her chief discomfort was in the right ear, in which there was a sensation of tension and stuffiness, and she was strongly impressed with the notion that the time had come for another cutting of this much-incised organ. Tests of the hearing showed that it was decidedly impaired on the right side, and they also localized the trouble in the middle ear. Examination with the aural speculum indicated that there was considerable liquid in the right tympanitic cavity, escape being prevented by occlusion of the eustachian tube on that side.

The anterior view of the fauces showed, at the first glance, that the soft palate was in a very abnormal condition. It was distorted by some internal pressure causing it to bulge forward. The quinsy points were conspicuous; showing through the mucous membrane were small nodules, pale, firm and hard, but not tender under the touch. The postnasal mirror showed a small clink behind the soft palate on the left side; but the right was filled up by a swelling projecting from the soft palate into the nasopharynx and preventing the finger from entering the postnasal space. This adventitious structure was firm and gave no sign of inflammation. Neither of the custachian eminences could be seen. Inflation by the Politzer method succeeded on the left side, but failed 
on the right. The custachian catheter passed readily along the nasal floor but, when its beak reached the soft palate, it met an obstruction and could not be advanced farther, nor conlel it be rotated.

These examinations proved that a tumor of considerable size was embedded in the soft palate, and from the signs given to sight and touch, combined with the clinical history I came to the conclusion that it was a chondroma, a judgment which was corroborated by the laboratory tests made sulisequently. I informed the patient of what had been fouml, telling her frankly and firmly that the best treatment, indeed the only proper treatment, was the entire removal of the tumor: that this course would give her good prospect of recovery; but that partial, half-way measures would be of no advantage. She took a sensible view of the matter and agreed to a prompt operation.

Just here let me say that I claim no credit for recognizing this neoplasm. It was in an accessible locality and could be seen distinctly by posterior rhinoscopy. Any laryngologist should have made himself familiar with its chief features on his first inspection of the throat. The puzzling questions are, why one specialist never looked for it and why another, after seeing it, told the patient that he did not know what it was and considered it best to let it alone.

Opcration and Results.-Two days later, October 2, the operation was performed under general anesthesia induced by ether. The right tonsil, which was somewliat morbid, was enucleated and the wound enlarged until it reached the level of the upper margin of the chondroma. This was dissected out. When its attachments had been severed, it was seen to have a lobular structure and came away in three pieces. Taken together these weighed a little more than an ounce. Bleeding was controlled by pressure, and the postoperative condition was normal.

The patient had a rapid convalesconce. Although the right ear received no topical treatment, hearing lecame alssolutely normal in fortyeight lours. The cough showed prompt improvement, and in ten days ceased entirely. Healing was complete on the sixteenth day, and there remained no disalislity of any description.

More than five months have now elapsed since the operation, and no morbid sequels have appeared. I saw the patient within a fortnight, and she was in excellent health and spirits. There can be no doult that the chondroma was the cause of all the varied symptoms from which she had suffered during six years and whose persistence threatened to bring her to invalidism.

\section{COMMENT}

I have given the history of this case thinking that it may prove both a caution and an incentive. We are all liable to err, and if oversights, such as those narrated, are frankly criticized, this is not done with a purpose to humiliate our colleagues, who appear to have been at fault, but in the hope that all of us may be stimulated to rencwed appreciation of the importance of thoroughness in searching for causation.

Only hy making a correct diagnosis and directing our treatment to the radical removal of the causes of disease can we do our full duty to our patients and attain that high professional ideal which combines wide knowledge of medical science with expert proficiency in the honored profession, which is our chosen vocation.

1906 Chestnut Street.

Practical Theories.-The practical effect of a belief is the real test of its soundness.-Froude.

\section{STERILIZATION OF SURGEONS' KNIVES AND SCISSORS BY HEATING IN LIQUID PETROLATUM * \\ Henky Lyman, M.D., Boston}

The subject of properly sterilizing knives and scissors has always given the surgeon more or less trouble. Boiling water or 70 per cent. alcohol, if applied long enough to be effective, inevitably destroys the edge, while the instruments so treated have a strong tendency to rust. It has been my practice for some time to sterilize ncedles which were to be used for drawing blood, by heating them in liquid petrolatum at $120 \mathrm{C}$. for twenty minutes. The needles may then be left for an indefinite length of time without danger of rusting, and are moreover coated with a film of oil which tends to prevent clotting. I soon noticed that needles so treated were peculiarly sharp and could be used and resterilized many times without losing their points. It occurred to me that the same method might well be employed on the scalpel and scissors of the surgeon with advantage, provided this heating did not affect the cutting qualities of the instruments. I thereforc sharpened a knife to a razor elge, heated it in the oil at $200 \mathrm{C}$. for over half an hour, and again tested the edge. It was exactly as sharp as before heating, and repeated experiments with other knives and with scissors gave the same result. The reason for this secms clear. Both boiling water and alcohol attack steel, thus ruining the edge, while liquid petrolatum, being exceedingly inert chemically, has no such effect.

'The first question which arises in our minds in this connection is, "Does oil, which may be considered iry heat, clestroy organisms as quickly at the same temperature as does boiling water?" Since no adequate references could be found in the literature, I was obliged to investigate this point myself. I was fortunate cnough to interest $\mathrm{Dr}$. Harold C. Ernest in this work, and the experiments were carried out under his supervision in the bacteriologic department of the Medical School of Harvard University.

The following technic was employed: Strips of tin, used to simulate scalpels, were rlipped into a culture of the organism to be investigated, dried, and dropped into a test tube containing about 10 c.c. of liquid petrolatum previously heated to the desired temperature in an oil bath. After the temperatute had been maintained for ten minutes, the strip was removed and firmly stroked on a slant of agar nutrient medium. For fear the organisms might have been washed off $i n$ this process, the oil was treated with sterile boullon, thoroughly stirred, allowed to settle, the bouillon pipetted off, and both the bouillon and the agar incubated. Numerous controls were run on cach batch of determinations.

It was found by this method that the Staphylococcus pyogenes-aureus and albus were killed in ten minutes at a temperature of $105 \mathrm{C}$., and that anthrax spores, possibly the hardest form of life to destroy of which we know, were killed in ten minutes at $150 \mathrm{C}$. The staphylococci were killed at $150 \mathrm{C}$. in fifteen seconds. If, therefore, the surgeon will keep his knives and scissors in an oil bath at $150 \mathrm{C}$. for ten minutes, their cutting qualitics will not be affected, they will not rust, and they will become the strongest link in the chain of asepsis, instead of being the weakest, as is the case under the usual method of sterilizing.

To obtain the best results, the oil used should have the following characteristics: it must be nonirritating, it must be chemically inert, it must be clear so that the instruments may be easily seen, and it must have the highest possible

\footnotetext{
* From the Huntington Menorial Hospital.
} 